\title{
Computational Mobility, Transportation, and Logistics
}

\author{
Natalia Kliewer · Jan Fabian Ehmke • \\ Dirk Christian Mattfeld
}

Published online: 3 April 2017

(C) Springer Fachmedien Wiesbaden 2017

This special issue focuses on the engineering of information systems that enable modern mobility, transportation and logistics services (MTL). In recent years, thanks to the ubiquitous availability of mobile sensors, huge amounts of operational data from MTL have become available. Furthermore, advancing automation of MTL enables data-driven, customer-oriented and innovative business models that lead to an increasing variety and competition of MTL. For instance, online retailers such as Amazon.com offer even same-day delivery within a time frame of 90 min today, which could not be realized without the support of smart, data-driven information systems for logistics services. Furthermore, to provide a car or bike whenever customers expect one, shared mobility services such as Car2Go or Flinkster depend on fully-automated support by information systems.

Next to the vast amount of increasing data of MTL, quantitative methods of service planning, service control, and service operations management need to be adapted and

Prof. Dr. N. Kliewer $(\bowtie)$

Department Wirtschaftsinformatik, Freie Universität Berlin, Garystraße 21, 14195 Berlin, Germany

e-mail: natalia.kliewer@fu-berlin.de

URL: http://www.wiwiss.fu-berlin.de/fachbereich/bwl/pwo/

kliewer/team/professorinnen/kliewer-natalia/index.html

Prof. Dr. J. F. Ehmke

Business Analytics, Europa-Universität Viadrina, Große

Scharrnstr. 59, 15230 Frankfurt (Oder), Germany

e-mail: ehmke@europa-uni.de

URL: https://www.wiwi.europa-uni.de/ehmke

Prof. Dr. D. C. Mattfeld

Institut für Wirtschaftsinformatik, Technische Universität Braunschweig, Mühlenpfordtstraße 23, 38106 Braunschweig, Germany

e-mail: d.mattfeld@tu-braunschweig.de

URL: https://www.tu-braunschweig.de/winfo/team/mattfeld extended to support innovative, automated MTL. However, it is challenging to analyze operational data and transform it into information that can be accessed by advanced planning methods. In addition to well-known efficiency objectives such as minimizing costs or time of operation, objectives of reliability and sustainability can now be considered in the planning and control of MTL. Processing of complex information needs to be considered in order to enable more reliable, sustainable and efficient planning and realization of MTL.

A special purpose of this application-oriented special issue is to shed light on quantitative methods that are core parts of information systems for MTL. We were especially interested in complex, quantitative methods of planning and control as well as applications of state of the art of data analysis procedures, such as methodological innovations from the areas of business analytics, operations research, simulation, revenue management, as well as dynamic and robust planning approaches. Examples of innovative integrated information systems for single as well as for several modes were also welcome.

This special issue consists of three research articles, a catchword article and an interview. We were able to select the research articles from a total of eleven submitted papers.

Gabriel Bazi, John El Khoury, and F. Jordan Srour focus on road infrastructure and present an information system that incorporates location data to optimize the routing of pavement data collection vehicles. In their paper, they examine the processes required to generate the list of roads for pavement testing, select appropriate hotels in the region of testing, and apply a mathematical model to determine a route of a pavement testing vehicle. Their experiments highlight significant cost savings associated with this method of roadway testing as opposed to the current, manual ad hoc practice. 
Kirsten Hoffmann, Udo Buscher, Janis Sebastian Neufeld, and Felix Tamke present a quantitative piece of work that focuses on the planning of the conductors shifts required to operate trains in public rail transportation. Arising from a practical problem in German rail passenger transport, they develop a prototype for a multi-period railway crew scheduling problem with attendance rates for conductors. Based on a business-analytics approach, they consider real-world data in quantitative planning methods. The prototype is evaluated with the help of a case study for three real-world transport networks. It is shown that the presented hybrid optimization approach is able to solve the problem more effectively and efficiently compared to conventional approaches from practice.

Contrasting the a priori perspective of the above papers, Marlin Ulmer, Leonard Heilig, and Stefan Voß discuss the value and challenges of real-time information in the dispatching of service vehicles. Since real-time information enables replanning at any point of time today, it is not obvious when replanning should be triggered. Frequent replanning may lead to efficient routing decisions due to vehicles' diversions from current routes, while less frequent replanning may enable effective assignments due to gained additional information. In their paper, they analyze and quantify the impact of exogenous customer requests, endogenous vehicle statuses, and replanning in fixed intervals for a dynamic vehicle routing problem with stochastic service requests. Computational experiments show under which conditions which trigger is advantageous.

Besides these three research contributions, this special issue also contains a BISE Catchword article by Christoph Willing, Tobias Brandt, and Dirk Neumann dealing with "Intermodal Mobility". The authors provide a well-structured overview of existing multi- and intermodal mobility solutions. They analyze the topic from an IS research perspective with special focus on the ways how the business and information systems engineering research community can contribute to the advancement of future mobility behavior.

The special issue is complemented with an interview of challenges and requirements of computational mobility, transportation and logistics in practice. The interview was given by Hanno Schülldorf (Deutsche Bahn AG, DB Analytics).

As guest editors of this special issue, we would like to thank the authors of all submitted papers for their work, and the reviewers for their timely and constructive contributions. 